\title{
Demonstration of Heat Resistant Fiber Bragg Grating Sensors Based on Femtosecond Laser Processing for Vibration Monitoring and Temperature Change
}

\author{
Akihiko Nishimura $^{* 1}$, Yusuke Takenaka ${ }^{* 2}$, Takehiro Furuyama ${ }^{* 2}$, Takuya Shimomura $^{* 3}$, \\ Takaya Terada ${ }^{* 3}$ and Hiroyuki Daido ${ }^{* 3}$ \\ *1 Japan Atomic Energy Agency, 8-1-7, Umebidai, Kizugawa, Kyoto, 619-0215 Japan \\ E-mail: nishimura.akihiko@jaea.go.jp \\ ${ }^{* 2}$ A-Tech, 65-20, Kizaki, Tsuruga, Fukui, 914-8585 Japan \\ *3 Japan Atomic Energy Agency , 65-20, Kizaki, Tsuruga, Fukui, 914-8585 Japan, 914-8585
}

\begin{abstract}
Heat resistant FBG sensors were developed by femtosecond laser processing to apply them to hightemperature- operated piping system of nuclear power plants. The FBG sensor was installed on the surface of a steel blade and a vibration test was conducted to detect the resonant vibration frequency of the vibrating blade. The FBG sensor had the heatproof performance at $600{ }^{\circ} \mathrm{C}$. A frequency stabilized sensing system using a tunable laser was tested for structural health monitoring in daily operation of nuclear power plants. The FBG sensor was installed on the surface of a steel blade for vibration induced strain measurements. Welding, brazing, soldering and noble metal powder adhesive were discussed for molding the FBG sensors. DOI: $10.2961 / \mathrm{jlmn} .2014 .03 .0007$
\end{abstract}

Keywords: Femtosecond laser processing, FBG sensor, heat resistant performance, strain measurement, tunable laser

\section{Introduction}

Developments of the new sensing system under harsh environmental conditions are now emerging from laser processing. It requires the advanced sensors that can run at high fidelity, and simultaneously needs the technique that can install them with practical ease.

Since the Noto peninsula earthquake along Sea of Japan in 2007, we have been developing the technologies related to heat resistant strain sensors by laser processing techniques $[1,2]$. In order to measure both temperature and strain, fiber Bragg grating (FBG) sensors were chosen. The application is aiming at the structural health monitoring for high temperature piping system of nuclear power plants (NPPs). The situation requires extraordinary durability such as radiation resistance and noise isolation caused by nuclear reaction or electro-magnetic pulse. In ITER projects, huge precious vacuum components were designed [3]. Here fast neutron damage will be more serious than NPPs [4]. To date, the FBG sensors made by femtosecond laser processing are the best candidate for such harsh conditions. The validities of FBG sensors were reviewed elsewhere [5], in which the FBG sensors would be ideally suited for advanced energy production systems as harsh environment sensing.

However, the present FBG-sensor-based reflection spectrum detection system in JAEA is now facing two principal problems: firstly, the rate at which the system can be scanned by combination of a broadband light source and a grating spectrometer is relatively low. When the system is detecting seismic vibrations of facilities due to ground motions, low repetitive scanning rate is not problematic be- cause the typical frequency is less than $10 \mathrm{~Hz}$. However, if we pay attentions to fluid dynamics of coolant flow in piping systems, much faster response on the system is required. Secondly, the FBG sensors need careful handling due to the fragility. The combination with fabric reinforcement and heatproof adhesive can provide one solution to protect fragile optical fiber in the sensor installation on the surface of piping material [6]. To get over the fragility, we are now developing the metal molding technique which can hold and fixe the FBG sensors. Some techniques using laser cladding or brazing are under development, depending on the operational temperature of facilities.

In his paper, from the view point of smart laser processing, a FBG-sensor-based reflection spectrum detection system was proposed. Some metal molding techniques using laser cladding were described. In each case of pressurized water reactors or fast neutron reactors, available techniques are described in the following chapters.

\section{FBG-sensor-based reflection spectrum detection system}

Figure 1 shows a block diagram of the FBG-sensor based reflection spectrum detection system which has potentially faster response. Here the laser system is a frequency stabilized continuous-wave tunable laser. Continuous, mode-hop-free wavelength sweeps was performed over its full $130 \mathrm{~nm}$ tuning range with $100 \mathrm{~nm} / \mathrm{s}$ sweep speed. The wavelength accuracy was suppressed to $<5 \mathrm{pm}$. An InGaAs photo-detector with $10 \mathrm{MHz}$ was used to detect the reflection spectrum from the FBG sensor. 


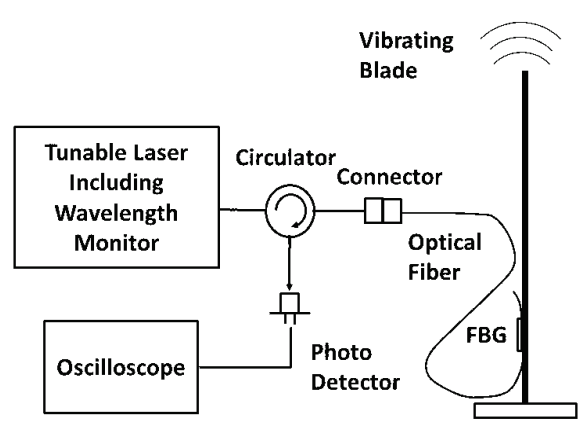

Fig. 1 Block diagram of FBG-sensor-based reflection spectrum detection system

The tunable laser including wavelength monitor function and an optical fiber processed with a FBG sensor were connected by an optical fiber circulator. We tried some ways how to mount a FBG sensor on a vibrating steel blade. At the first time, a FBG sensor was fixed on a $0.3 \mathrm{~mm}$ thickness steel plate with heatproof epoxy glue. Preheating for epoxy glue was done at $230{ }^{\circ} \mathrm{C}$ for 8 hours. Then it could work at the maximum temperature of $400{ }^{\circ} \mathrm{C}$. A steel blade with $47 \mathrm{~cm}$ length, $7 \mathrm{~cm}$ width and $4 \mathrm{~mm}$ thickness was prepared to be set perpendicularly to a $25 \mathrm{~A}$ flange by arc welding. Then the steel plate with a FBG sensor and the vibrating steel blade were soldered up. The position of the FBG sensor was $5 \mathrm{~cm}$ away from the flange. The reflection laser light coming back from the FBG sensor was monitored by a digital oscilloscope. The FBG sensor was heated by heat conduction behind the vibrating blade.

\section{Fabrication of the FBG sensors}

The FBG sensors were fabricated by femtosecond laser processing. The details were described elsewhere $[2,6]$. The outline was shown in the following procedure. A CPA laser with a fiber femtosecond oscillator generated 100 fs laser pulses. The pulse energy was precisely controlled for the processing by an object lens into a fiber core, being limited to $10 \mu \mathrm{J}$ at $100 \mathrm{~Hz}$ repetition. At the output of the amplifier, an air-gap polarizer and $1 / 2 \lambda$ wave plate were put in to attenuate the laser pulse energy in order to reduce the output laser energy of $1 \mu \mathrm{J}$ or less. The laser pulses were focused into the center of the fiber core which was mounted on a translation stage.

Figure 2 shows a picture of the fiber core image of the FBG structure by a phase contrast microscope. Femtosecond laser pulses were delivered into a microscope and focused on the fiber core with dot spot of 1-2 $\mu \mathrm{m}$ diameter. Observing the laser processing through the microscope, we confirmed that laser focused dots lined up perfectly along the center of the fiber core. The dot diameter in a fiber core was always smaller than Gaussian laser intensity distribution due to the nonlinear effect. Super-white continuum flash was observed on a CRT monitor of the microscope. Rotating the fiber at 90 degree after laser processing, we find these dots formed series of extended longitudinal cylinder. It seemed to be a comb laid inside a fiber core and worked as a reflection grating. Laser light for reflection detection travels along the fiber core from right side to left side.

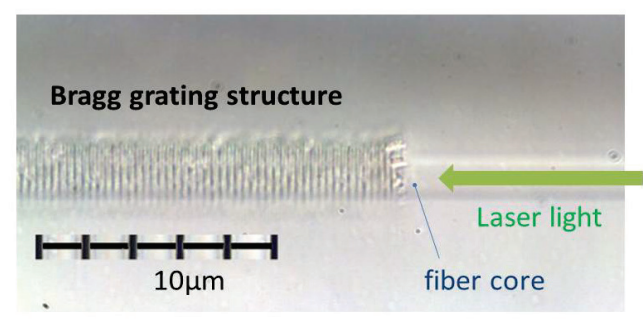

Fig. 2 Picture of FBG structure made in the fiber core

Figure 3(a) (b) shows the comparison of reflection spectra of two FBG sensors having dot-number dependent different peak widths. These spectra were processed by point by point writing technique using femtosecond laser pulses. We understand that the peak width is inversely proportional to the dot number, comparing the result of 100 dots and that of 250 dots. However, some satellite peaks surrounding the center peak at $1553 \mathrm{~nm}$ were enhanced with increasing the dot number. Generally speaking, an isolated sharp peak on the FBG spectra is preferred in order to achieve high stability of peak wavelength tracking. On the other hand, when the detection system activating a frequency stabilized wavelength, a broaden line width seems more adaptable.

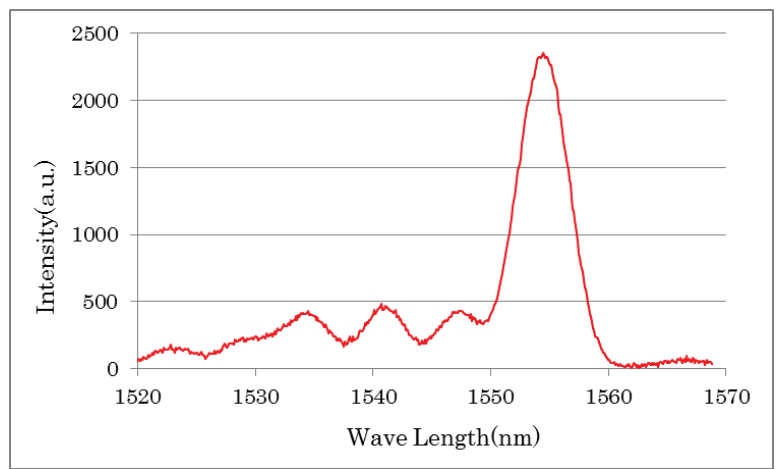

(a)

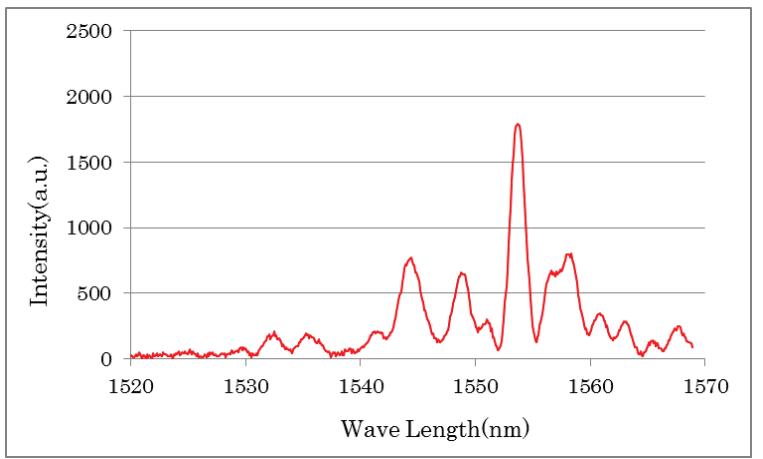

(b)

Fig. 3 Comparison of reflection spectra of FBG sensors having different dot numbers. (a) dot number 100, (b) dot number 250

Using point by point writing technique, it is easy to fabricate custom demand FBG sensors. In JAEA, we could use the apodizing mask or the filtering window which can control laser pulse energy in order to clean up the spectrum. This is one way to reach the goal. It is well known in data 
analyzing technique that the discrete-time Fourier transform of the rectangular window shows distortion in spectrum domain [7]. The laser induced dot sequence must be limited to a finite length to generate Bragg interference. It causes build-up the satellite peaks. Instead of the rectangular window function, the Hanning/Hamming window could reduce the intensity of those peaks. However, this method cannot be completely free from mechanical error driven by the stage motion. Thus the interference processing technique is inherently and potentially superior to the point by point technique. As a femtosecond laser can generate intense pulse energy for FBG sensor processing, mass production of FBG sensor is also feasible.

The maximum reflectivity occurs at the Bragg wavelength $\lambda_{B}$ which is given by the equation; $\lambda_{B}=2 n_{\text {eff }} \Lambda / \mathrm{m}$. Where $n_{\text {eff }}$ is the effective refractive index of the mode propagating in the fiber, $\Lambda$ is the FBG pitch period and $\mathrm{m}$ is the FBG harmonic order. This relation shows that the reflected wavelength $\lambda_{\mathrm{B}}$ is affected by any variation in the physical or mechanical properties of the Bragg grating region. Practically to say, in this study, temperature change and strain induction are representative properties for secondary cooling loop. In primary containment vessels or primary cooling loops, radiation induced effects could be added to them. This is expressed in the following equation, where the first term gives the effect of temperature and the second describes the effect of strain [8].

$$
\Delta \lambda_{\mathrm{B}}=\lambda_{\mathrm{B}}(\alpha+\xi) \Delta \mathrm{T}+\lambda_{\mathrm{B}}(1-\rho \alpha) \Delta \varepsilon
$$

Where $\alpha$ and $\xi$ and $\rho \alpha$ are respectively the thermal expansion and thermo-optic coefficients and photoelastic coefficient of the silica fiber, and $\Delta \mathrm{T}$ is the temperature change and $\Delta \varepsilon$ is the strain change. For a typical grating written in a silica fiber and with $\lambda_{\mathrm{B}} \approx 1550 \mathrm{~nm}$, sensitivities to temperature and strain are approximately $10 \mathrm{pm} /{ }^{\circ} \mathrm{C}$ and $1.2 \mathrm{pm} / \mu \varepsilon$ respectively.

\section{Installation of the FBG sensors}

How to install the FBG sensors on the surface of steel surface is a troublesome issue. To make the best use of the heat resistant characteristic, we demonstrated two ways to embed the FBG sensor by metal mold. One way was soldering or brazing. In the case of soldering, a filler metal was a kind of alloy based on lead or tin to be heated to the melting point around $200{ }^{\circ} \mathrm{C}$. To get higher temperature durability, silver based blazing could be applied to embed the FBG sensor, which heats the FBG sensor ranging from $600 \sim 800{ }^{\circ} \mathrm{C}$. Prior to brazing, the brazing surface needs abrasive or mechanical cleaning. In the case of atmosphere environment, it also requires brazing flux/paste in preparation. To heat the surface in the vicinity of the optical fiber, we used a homemade handy laser torch. The heat source was a CW laser with $300 \mathrm{~W}$. The drawback of brazing is erosion of the optical fiber caused by brazing flux/paste.

The other way was welding/cladding to use a filler wire of $\mathrm{Cr}$ contained iron, heating the FBG sensor up to iron melting point. A groove was processed to the surface of a SUS metal plate with a rotating grindstone. We used a Quasi-CW laser to make a cladding with a filler wire along the groove. The optical fiber set under molding metal before- hand was heated by the laser pulses with 10 joule energy and $10 \mathrm{~ms}$ duration, partially to be soaked in a metal mold pool. A series of weld pool formed a sealing clad on the groove. The FBG sensor was buried at $2 \mathrm{~mm}$ depth over the length of $1 \mathrm{~cm}$. On the reflection spectra, the wavelength shift of $6 \mathrm{~nm}$ was detected during heating process. The FBG sensor was able to use detection of abrupt temperature change of a steel plate caused by thermal shock with pulsed laser heating.

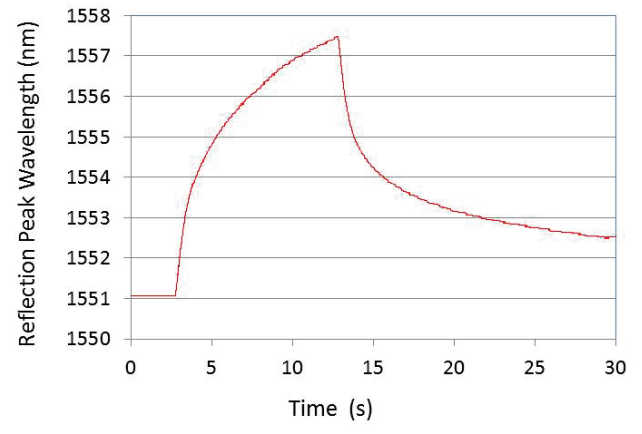

Fig. 4 Time dependence of reflection peak wavelength of FBG sensors observed for laser heating of 10 second duration

Figure 4 shows the peak wavelength shift of FBG sensor due to laser heating during 10 seconds. At the laser power of $320 \mathrm{~W}$, over $6 \mathrm{~nm}$ wavelength shift of a reflection peak from $1551 \mathrm{~nm}$ was detected during heating process. The FBG sensor was tolerated even 17 times periodic heating. No degradation was observed. This reflection peak shift was equivalent to $600{ }^{\circ} \mathrm{C}$ and more when it was converted to temperature. The FBG sensor embedded in a SUS plate showed a good reproducibility. It was proved that frequent vibration of the steel blade shown in Fig. 1 was successfully detected as a result of the proper installation of the FBG sensor.

A vibration test was conducted to evaluate the response of the FBG sensor metal mold using frequency resonant vibration of a steel blade. Figure 5 shows an example of periodic reflection intensity modulation caused by a FBG sensor. Here, the vibration of a steel blade was clearly detected. The resonant frequency was $18 \mathrm{~Hz}$ of the vibrating steel blade by FFT analyzing. This frequency is high enough in comparison with seismic motion of the nuclear facilities.

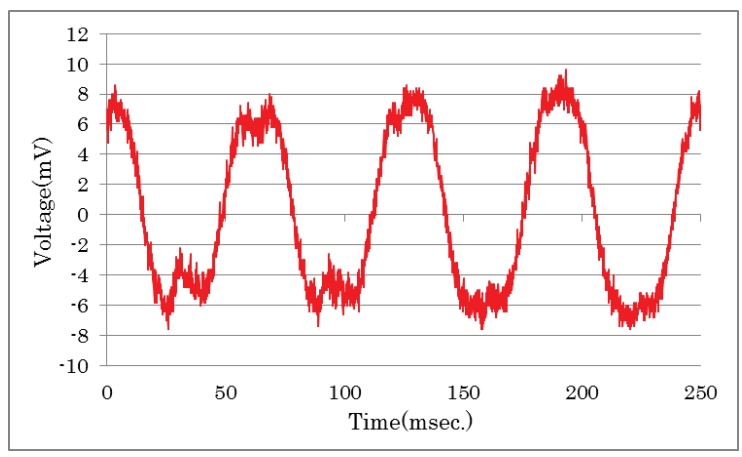

Fig. 5 Periodic reflection signal intensity change of the FBG sensor caused by the vibration of a steel blade 


\section{Discussions}

One of the most important parameters how to deploy the FBG sensors to engineering plants or nuclear facilities is the operational temperature. Outlet coolant temperature decides the whole efficiency of power plants. The highest category is a high temperature gas reactor. Helium gas temperature of a high temperature gas reactor reaches over $900{ }^{\circ} \mathrm{C}[9]$. This value exceeds the melting point of brazing metals. Welding iron based clad metals is only way to hold the molding stable. The next harsh category is a sodium cooled reactor. Liquid sodium temperature in the primary cooling loop reaches $550{ }^{\circ} \mathrm{C}$, which may cause creep fatigue in structural materials such as SUS316 [10]. This value surely exceeds the limit temperature of heat proof epoxy adhesive and the melting point of soft soldering alloys. Noble metal power based adhesive or ceramics powder based adhesive could work. Brazing or hard soldering using copper or silver based metal rod/powder is also available at this temperature. The moderate category is including major power plants such as boiling water reactors, pressurized water reactors and so on. The operational temperature lies in the range of $250 \sim 350{ }^{\circ} \mathrm{C}$.

An optical fiber has a primary coating in general. The primary coating protects the clad fiber against mechanical damage and acts as a barrier to lateral forces. It also serves as a barrier to moisture. Without the coating, a clad fiber is easy to be broken due to its poor handling and durability. The surface properties of the coating must be carefully controlled, to allow good adhesion under laser cladding temperature in a metal mold. Poly-imide is representative resin for clad coating, which can be commercially available, while at the same time allowing for good wettability providing a condition for impregnation with molten metal liquid in a few seconds during cooling period of laser cladding. In the condition of $200{ }^{\circ} \mathrm{C}$ or less, the optical fiber diameter was kept constant and no color changed on the fiber surface was found. Over $250{ }^{\circ} \mathrm{C}$, slight degradation began and the surface color changed from copper torn red to deep tuning brown. Heating the optical fiber ranging from 500 to $550{ }^{\circ} \mathrm{C}$, we found that the primary coating was gradually sublimed after carbonization.

To the practical point of view for the installation of FBG sensors on nuclear facilities, metal adhesive might be the best candidate. Commonly, metal powder based adhesive consists of water, binders, and fine particles of either silver or gold. After being preheated at $200{ }^{\circ} \mathrm{C}$, it can be used up to $900{ }^{\circ} \mathrm{C}$. In the case of a steel plate, it was useful to electroplate copper on the surface in advance of applying the metal adhesive. In nuclear facilities, workers will be forced to do delicate works with difficulty to attach FBG sensors, wearing a radiation protection suit and gloves. When they install the FBG sensors on the surface of coolant pipelines, there will be large difference with heat capacity between the metal mold and the surface. To get strong adhesion, they would have to heat the surface of pipelines rather than the metal mold. So they must heat the FBG mold due to heat conduction, maintaining the surface of lines slightly hotter than the FBG mold. A specially designed compact heater or a handy laser torch may be useful for spot heating. Gradual heat conduction would enhance to evaporate liquid binder. It would finally make fine metal powder sintered tightly. Then the metal mold can hold the FBG sensors in its inside.

Two significant events are causing the waning of nuclear power in Japan now. The first significant event was the nuclear accident that occurred at the Fukushima Daiichi NPP in 2011. And the second significant event would be the fact that restarting power plants have degrading structural materials unable to be replaced. They can be said as sudden death and slow death. Seismic monitoring on the pipeline by the heat resistant FBG sensors can contribute to the severe present situation in Japan.

\section{Conclusion}

The heat resistant FBG sensors were fabricated by femtosecond laser processing. To use the sensors as strain gauges for nuclear power plants, installation techniques by metal molding were developed. Laser cladding with a filler wire was successfully embedded the FBG sensor on a SUS plate. The frequency of a vibrating steel blade was monitored by the FBG sensor. Brazing or metal powder adhesive was also applicable to install them on the surface of pipelines.

\section{Acknowledgments}

The authors appreciated Prof. T. Kobayashi of Fukui Univ. for his valuable comments to process the FBG sensor. The authors also wish to acknowledge about heating tests with a large scale pipeline which is under planning in collaboration with KUMAGAI-GUMI, Co. ltd.

\section{References}

[1] Y. Shimada, T. Masuzumi, A. Nishimura, M. Todi and K. Tsukimori, 2008 Annual Meeting of the Atomic Energy Society of Japan, March 26-28, Osaka, (2008) J25. (in Japanese)

[2] Y. Shimada, A. Nishimura, et al., Journal of Laser Micro/Nanoengineering, 5, (2010) 99.

[3] K. Ioki, et al., Fusion Engineering Design, 87, (2012) 828.

[4] A. F. Fernandez, et al., IEEE Trans. Nucl. Sci., 48(5), (2001) 1708

[5] S. J. Mihailov, Sensors, 12(2), (2012) 1898.

[6] Y. Shimada, and A. Nishimura, Journal of Laser Micro/Nanoengineering, 8, (2013) 110.

[7] M. Hino: "SPEKUTORU KAISEKI (Spectrum Analysis) " (Publisher, Asakura, 1977) Chapter 10. (Books)

[8] Page 1900 of ref. [5].

[9] D. Tochio and S. Nakagawa, J. Nucl. Sci. Technol., 48(11), (2011) 1361.

[10] T. Asayama, S. Takaya, Y. Nagae, M. Ando and K. Tsukimori, Procedia Engineering 55 (2013) 309.

(Received: April 13, 2014, Accepted: August 15, 2014) 